\title{
DMP IN STRONGLY MINIMAL SETS
}

\author{
ASSAF HASSON* AND EHUD HRUSHIVSKI ${ }^{\dagger}$
}

\begin{abstract}
We construct a strongly minimal set which is not a finite cover of one with DMP. We also prove that for a strongly minimal theory $T$ generic automorphisms exist iff $T$ has DMP, thus proving a conjecture of Kikyo and Pillay.
\end{abstract}

Recall that a strongly minimal theory $T$ has the Definable Multiplicity Property (DMP) if for all natural $k, m$ and $\varphi(\bar{x}, \bar{b})$ of rank $k$, multiplicity $m$ there exists a formula $\theta \in t p(\bar{b})$ such that for all $\bar{b}^{\prime} \models \theta, r k\left\{\varphi\left(\bar{x}, \bar{b}^{\prime}\right)\right\}=k$ and $\operatorname{mult}\left\{\varphi\left(\bar{x}, \bar{b}^{\prime}\right)\right\}=m$. This definition was introduced in [2], where it was asked whether every strongly minimal set is a finite cover of one with DMP, i.e. whether for every strongly minimal $T$ there exists a definable equivalence relation $E$ with finite classes such that $T / E$ has DMP.

In [4] Kikyo and Pillay prove that a strongly minimal $T$ (with quantifier elimination) which is a finite cover of a theory with DMP has generic automorphisms (i.e. the theory $T \cup\{\sigma$ is an automorphism $\}$ has a model companion) iff $T$ itself has DMP. They conjectured that the same is true of any strongly minimal theory.

In the first section of this paper we construct a strongly minimal set which is not a finite cover of one with DMP. In the second part we prove Kikyo and Pillay's conjecture.

\section{A strongly Minimal Set With no DMP}

In this section we slightly change the construction of [3] to obtain:

Theorem 1.1. There exists a strongly minimal theory $T$ such that for every definable infinite equivalence relation $E$ with finite classes, $T / E$ does not have DMP.

It seems that our construction methods can be generalized, but for the sake of simplicity and clarity we will stick to the simplest example. We will go very briefly through most of the definitions and standard statements, as they are practically similar to the corresponding ones in [3].

Let $L:=\left\{S, R_{i}, a_{i}\right\}_{i<\omega}$ be a language consisting of one ternary relation $S$, countably many binary relations $R_{i}$ and a set of constants $\left\{a_{i}\right\}_{i<\omega}$. For any $L$ structure $A$ we denote $\hat{A}:=A \backslash\left\{a_{i}\right\}_{i<\omega}$ and say that $A$ is $L$-finite if $\hat{A}$ is finite. For an $L$-finite structure $A$ we denote $R(A):=\left\{z \in A^{3}: A \models S(z)\right\}, r(A)=|R(A)|$ and $d_{0}(A)=|\hat{A}|-r(A)$. Let $T_{\forall}$ be the following theory:

(1) $d_{0}(A) \geq 0$ for every $L$-finite structure $A$.

(2) $R_{i}\left(z_{1}, z_{2}, z_{3}\right) \Rightarrow z_{1}=a_{i} \wedge S\left(a_{i}, z_{2}, z_{3}\right)$.

\footnotetext{
* Supported in part by the EPSRC grant no. EP C52800X 1.

†Supported in part by Israel Science Foundation Grant no. 244/03.
} 
Let $\mathcal{C}$ be the class of $L$-finite models of $T_{\forall}$. For $B_{1}, B_{2} \in \mathcal{C}$ and $A=B_{1} \cap B_{2}$ we define the free join of $B_{1}$ with $B_{2}$ over $A$, denoted $B_{1} \oplus_{A} B_{2}$ as the $L$-structure whose universe is $B_{1} \coprod_{A} B_{2}$ and whose relations are precisely those appearing already in $B_{1}$ or $B_{2}$. As usual we set for $A \subseteq B \in \mathcal{C}$

$$
d_{B}(A)=\min \left\{d_{0}(C): A \subseteq C \subseteq B\right\}
$$

and $A \leq B$ if $d_{B}(A)=d_{0}(A)$, in which case we also say that $A$ is self-sufficient in $B$. If $\bar{A} \leq B$ and $d_{0}(B / A):=d_{0}(A \cup B)-d_{0}(A)=0$ we say that $B$ is prealgebraic over $B$. It is simply pre-algebraic if $d_{0}\left(B^{\prime} / A\right)>0$ for all $A \subsetneq B^{\prime} \subsetneq B$. If in addition $A$ is minimal such that $B / A$ is simply pre-algebraic we say that $B / A$ is simply minimally pre-algebraic. It is standard to check that $\leq$ is transitive.

The following is easy and will be used without reference. The proof is left as an exercise:

Claim 1.2. Let $F \subseteq A, B \subseteq N \models T_{\forall}$. If $B / F$ is minimally simply pre-algebraic, $A \leq N$ and $B^{\prime} \in \operatorname{atp}(B / F)^{N}$ then either $B^{\prime} \cap A=F$ or $B^{\prime} \subseteq A$.

Let $B / A$ be minimally simply pre-algebraic. Let $\bar{a}:=\bigwedge_{i=1}^{3} \pi_{i}(R(B)) \cap A$ for $\pi_{i}$ the natural projection onto the $i^{\text {th }}$ coordinate. Let $\psi_{B / A}(y) \in \operatorname{atp}(\bar{a})$ isolate the type, and $\phi_{B / A}(x, y)$ isolate $\operatorname{atp}_{S}(\hat{B} / \bar{a})$. Let

$$
\mathcal{A}:=\{(B, A): B / A \text { is minimally simply algebraic }\}
$$

We fix a function $\mu: \mathcal{A} \rightarrow \mathbb{N}$ with the following properties:

(1) If $(B, A),\left(B^{\prime}, A^{\prime}\right) \in \mathcal{A}$ and $A^{\prime} \models \psi_{B / A}, B^{\prime} \models \phi_{B / A}\left(x, A^{\prime}\right)$ then $\mu(B, A)=$ $\mu\left(B^{\prime}, A^{\prime}\right)$.

(2) If $(B, A) \in \mathcal{A}$ and $\tau$ is a permutation on $\{1, \ldots,|B|\}$ fixing $A$ setwise then $\mu(\tau(B, A))=\mu(B, A)$

(3) $\mu(B / A) \geq \max \left\{d_{0}(A), 2\right\}$.

If $B / A$ is simply pre-algebraic (but not necessarily minimally so) we may abuse the notation and write $\mu(B, A)$ meaning $\mu(B, F)$ for the unique $F \subseteq A$ such that $B / F$ is minimally simply pre-algebraic. In addition, by (2) above, there will not be any ambiguity in referring - as we will - to $\mu(B, A)$ without specifying an enumeration of $B$ and $A$.

Remark 1.3. The requirement that $\mu(B / A) \geq d_{0}(A)$ is not used explicitly in the text, but appears implicitly in the proof of Lemma 1.5 below. The requirement that $\mu(B / A) \geq 2$ does not appear in the original construction and will be only needed to obtain Lemma 1.15.

Definition 1.4. We will denote $\mathcal{C}_{\mu} \subseteq \mathcal{C}$ the sub-class of all L-finite $M \models T_{\forall}$ in which there are at most $\mu(B / A)$ pairwise disjoint realizations of atp $p_{S}(B / A)$ for any fixed $A \subseteq M$ and $A \leq B \in \mathcal{C}$ minimally simply-algebraic.

The following is Lemma 3 of [3]. Both the statement and proof remain unaltered:

Lemma 1.5. Suppose that $A, B_{1}, B_{2} \in \mathcal{C}_{\mu}$ are such that $A \subseteq B_{1}$ and $B_{1} / A$ is simply pre-algebraic. Assume for simplicity that $A=B_{1} \cap B_{2}$ and let $E=B_{1} \oplus_{A} B_{2}$ then one of the following is true:

(1) $E \in \mathcal{C}_{\mu}$. 
(2) There exists $F \subseteq A$ such that $B_{1} / F$ is minimally simply pre-algebraic and disjoint $C_{1}, \ldots, C_{r} \subseteq B_{2}$ such that atp $\left(C_{i} / F\right)=\operatorname{atp}_{S}\left(B_{1} \backslash F / F\right)$ and $r=\mu(B / F)$.

(3) There exists $X \subseteq B_{2}$ such that $X \cap A \not \leq X$ and $B_{1}$ contains an $S$-isomorphic copy of $X$.

Concerning the adaptation of the proof to the present context we note only that since $\mu$ depends only on the $S$-structure of $B / A$ the relations $R_{i}$ can be ignored for the purpose of this lemma. The two differences with respect to the original proof are that our structures are not finite (since they all contain the constants) and that $d_{0}$ is slightly changed. As both $B_{1}$ and $B_{2}$ are $L$-finite there is only a finite set of constants $C$ which contributes to the $S$-type of $B_{1} \cup B_{2}$, so we may restrict our attention to $\hat{E}:=\hat{B}_{1} \oplus_{\hat{A} C} \hat{B}_{2}$, which eliminates the first difference with the original proof. Requiring that (keeping the notation in the statement of the lemma) $C \cap C_{i}=\emptyset$ and that $X \subseteq \hat{B}_{2}$ eliminate the second difference.

In [3] self-sufficient amalgamation was an almost immediate corollary of (the analogue of) Lemma 1.5. In the present case this is clearly not enough, as the (quantifier free) $S$-type of any tuple does not isolate its (quantifier free) type. To obtain the self-sufficient amalgamation we add some more restrictions on our function $\mu$ and on the class $\mathcal{C}_{\mu}$.

Notation 1.6. Let $B \supseteq A$ be such that $|B \backslash A|<\infty$. We set

$$
c(B / A):=\left|S(B) \cap\left(A \times B^{2} \backslash A^{2}\right)\right|
$$

and

$$
\begin{gathered}
C o(B / A):=S(B) \cap\left(\left(\bigcup_{i<\omega} a_{i}\right) \times B^{2} \backslash A^{2}\right) \\
\operatorname{co}(B / A)=|C o(B / A)| .
\end{gathered}
$$

Remark 1.7. Let $B / A$ be minimally simply algebraic and $A^{\prime} \equiv_{S} A$. For any $B^{\prime}$ such that atp ${ }_{S}\left(B^{\prime}, A^{\prime}\right)=\operatorname{atp}_{S}(B, A)$ there are precisely $2^{\text {coo }\left(B^{\prime} / A^{\prime}\right)}$ complete atomic $L$-types $p_{i} \supseteq \operatorname{atp}_{S}\left(B^{\prime}, A^{\prime}\right) \cup a t p_{L}\left(A^{\prime}\right)$.

Proof. $p_{i}$ as above will be determined by the $R_{i}$ relations it implies. By $T_{\forall}(2)$ this consists in, for each $s \in C o(B / A)$, deciding whether $\models R_{\pi_{1}(s)}(s)$ or $\models \neg R_{\pi_{1}(s)}(s)$ $\left(\pi_{1}\right.$ the projection on the first coordinate). Since the choices are independent the remark follows.

Now we require that $2^{c(B / A)} \mid \mu(B / A)$ and define $\hat{\mathcal{C}}_{\mu} \subseteq \mathcal{C}_{\mu}$ by requiring that for every simply minimally algebraic $B / A$ and any $A \subseteq M \in \hat{\mathcal{C}}_{\mu}$ there are at most $\mu(B / A) / 2^{c o(B / A)}$ disjoint realizations of $\operatorname{atp}(B / A)$ in $M$. We can now prove:

Lemma 1.8 (Self-sufficient amalgamation). Suppose that $A, A_{1}, A_{2} \in \hat{\mathcal{C}}_{\mu}, A \leq A_{i}$. Then there exists $E \in \hat{\mathcal{C}}_{\mu}$ and embeddings $f_{i}: A_{i} \hookrightarrow E$ such that $f_{1} \uparrow_{A}=f_{2} \uparrow_{A}$ and $f_{i}\left(A_{i}\right) \leq E$.

Proof. The proof is easily adapted from [3]. We give the details for completeness. We proceed by induction on $\left|A_{1}-A\right|+\left|A_{2}-A\right|$. Let $d=d_{A_{i}}(A)=d_{0}(A)$. If there existed some $A \subsetneq X \subsetneq A_{1}$ such that $d_{0}(X)=d$ then $X \leq A_{i}$ and using the induction hypothesis twice we get the desired result.

If $d_{0}\left(A_{1}\right)>d$ and there is no $X$ as above, then there must be some $b \in A_{1} \backslash A$ such that $d(b / A)=1$ (in fact, any $b$ will do). It is immediate that in that case $A_{2} \cup\{b\} \in \hat{\mathcal{C}}_{\mu}$ and that $A \cup\{b\} \leq A_{i} b$, so that we can again proceed by induction. 
It remains to deal with the case that $A_{1} / A$ is simply pre-algebraic, so it is minimally simply pre-algebraic over some $C \subseteq A$. Since $A \leq A_{2}$ either (1) or (2) of Lemma 1.5 must hold (as (3) would contradict $A \leq A_{2}$ ). If (2) is the case then $\operatorname{atp}_{S}\left(A_{1} / A\right)$ is realized $\mu\left(A_{1} / C\right)$ times disjointly in $A_{2}$. By Remark 1.7 this can only happen if among them are $\mu\left(A_{1} / C\right) / 2^{c o\left(A_{1} / C\right)}$ ) realizations of $\operatorname{atp}\left(A_{1} / A\right)$. Because $A \leq A_{2}$ the minimality of $A_{1} / C$ implies that each such realization is either contained in $A$ or freely joint with $A$ over $C$. Since $A_{1} \in \hat{\mathcal{C}}_{\mu}$ the former case cannot happen. Hence there is a copy of $A_{1} \backslash A$ in $A_{2}$ freely joint with $A$ over $C$ and it is easy to check that we can identify $A_{1} \backslash A$ with this copy of itself.

Finally, if (1) is the case then the free join of $A_{1}$ with $A_{2}$ over $A$ contains at most $\mu\left(A_{1} / C\right)$ disjoint copies of $\operatorname{atp}_{S}\left(A_{1} / A\right)$. If no more than $\mu\left(A_{1} / C\right) / 2^{c o\left(A_{1} / C\right)}$ realize $\operatorname{atp}\left(A_{1} / C\right)$ we are done. Otherwise by the exact same argument, one of them must be disjoint form $A$ and contained in $A_{2}$ and we can identify $A_{1} \backslash A$ with this image of itself.

Corollary 1.9. There exists a (countable) structure $\mathcal{U}$ universal and homogeneous with respect to $\left(\hat{\mathcal{C}}_{\mu}, \leq\right)$, i.e. $\emptyset \leq \mathcal{U}$ and for every finite $A \leq U$ and $A \leq B \in \hat{\mathcal{C}}_{\mu}$ (finite) there exists an embedding $f: B \hookrightarrow{ }_{A} \mathcal{U}$ such that $f(B) \leq \mathcal{U}$.

We are now ready to give the axiomatization of the strongly minimal set we are aiming at. To keep the axiomatization simple we will use the following notation:

For $(B, A) \in \mathcal{A} \phi_{B / A}(x, y)$ be as in the definition of the function $\mu$ and set $n=|B \backslash A|$ for $m \in \mathbb{N}$ we define

$$
\theta_{B / A}^{m}\left(x_{1,1}, \ldots x_{m, n}, A\right):=\bigwedge_{j_{1} \leq j_{2}, i_{1} \neq i_{2}} x_{i_{1}, j_{1}} \neq x_{i_{2}, j_{2}} \wedge \bigwedge_{i=1}^{m} \phi_{B / A}\left(\bar{x}_{i}, A\right)
$$

where $\bar{x}_{i}:=\left(x_{i, 1}, \ldots, x_{i, n}\right)$.

Define $T_{\mu}$ to be:

- $T_{\forall}$

- For every model $M$ and $L$-finite substructure $B \subseteq M, B \in \hat{\mathcal{C}}_{\mu}$.

- For every model $M$, any $L$-finite $B \subseteq M$ and $B \leq C \in \hat{\mathcal{C}}_{\mu}$ such that $C / B$ is minimally simply pre-algebraic and such that $X \cap B \leq X$ whenever $X \subset M$ realizes an atomic type realized in $C$ there are $\mu(C / B)$ solutions of $\operatorname{atp}_{S}(C / B)$ which are pairwise disjoint and any other solution of $\operatorname{atp}_{S}(C / B)$ meets at least one of them. Formally, if the assumptions of the statement hold then:

$$
M \models\left(\exists \bar{x}_{1}, \ldots \bar{x}_{r}\right) \theta_{C / B}^{m}\left(\bar{x}_{1}, \ldots \bar{x}_{r}, B\right) \wedge\left(\neg \exists \bar{x}_{0}, \ldots \bar{x}_{r}\right) \theta_{C / B}^{m+1}\left(\bar{x}_{0}, \ldots \bar{x}_{r}, B\right)
$$

for $r=\mu(C / B)$.

- For every $k \in \mathbb{N}$, every fixed set of constants $\bar{a}:=\left\{a_{i_{1}}, \ldots, a_{i_{k}}\right\}$ every $M, B, C$ as above and such that $\bar{a} \subseteq C o(C / B)$ given $\mu(C / B)$ solutions of $\operatorname{atp}_{S}(C / B)$ as above there are precisely $\mu(C / B) / 2^{|\bar{a}|}$ among them satisfying each of the $2^{|\bar{a}|}$ completions of $\operatorname{atp}_{S}(C / B)$ to a complete atomic type in $S \cup\left\{\bar{a} \cup R_{i_{1}}, \ldots, R_{i_{k}}\right\}$.

The last axiom scheme in the definition of $T_{\mu}$ introduces the main novelty of the present construction with respect to the one in [3]. In the original construction, if $B / A$ is minimally simply algebraic its atomic type is isolated uniformly in $A$. This is not the case in the present situation. A single axiom of this last axiom scheme (for one q.f.- $S$-type of $B$ ) cannot cover all possible $L$-types of $B$. The new 
point is that other axioms, referring to specializations of $B$ also, precisely fill in the gaps left by the axiom referring to $B$ itself. This relies heavily on the fact that we have a precise count of the number of disjoint realizations of $\phi_{C / B}(x, y)$ for all $y \models \psi_{C / B}(y)$, with the count independent of $y$.

Claim 1.10. $T_{\mu}$ is first order.

Proof. $d_{0} \geq 0$ can be stated as:

For every finite subset $C$ of constants and any disjunction of atomic $S$-formulas $\phi(\bar{x}, \bar{y})$ with more than $|\bar{x}|$ distinct literals of the form $S\left(x_{i_{1}}, x_{i_{2}}, x_{i_{3}}\right)$ with $1 \leq i_{1} \leq$ $i_{2} \leq i_{3} \leq|x|$, an axiom $\left(\bigwedge_{i \neq j} x_{i} \neq x_{j}\right) \rightarrow \neg \phi(\bar{x}, \bar{c})$ for any enumeration $\bar{c}$ of $C$.

So $T_{\forall}$ is first order. The second axiom scheme (modulo $T_{\forall}$ ) simply bounds the number of solutions of certain equations, so is clearly first order. By condition (1) in the definition of $\mu$ the last two axiom schemes are given in such a way that every instance of them is already stated in first order way.

Remark 1.11. Note that (once the consistency of $T_{\mu}$ is verified, if $B \leq M \models T_{\mu}$ and $(C, B) \in \mathcal{A}$ then we can strengthen the conclusion in the third axiom scheme. In that case we can actually state that there are $\mu(C / B)$ solutions of atp $p_{S}(C / B)$ in $M$ which are all pairwise disjoint and such that any other solution of atp $p_{S}(C / B)$ is a permutation of one of them.

With this in hand the following claim is an immediate corollary of self-sufficient amalgamation (see e.g. Section 3 of [3] for more details) once we show that $T_{\mu}$ is consistent:

Claim 1.12. Let $M_{1}, M_{2}$ be saturated models of $T_{\mu}$ of the same cardinality and $A_{i} \leq$ $M_{i},\left|A_{i}\right|<\left|M_{i}\right|$ then any isomorphism $f: A_{1} \rightarrow A_{2}$ extends to an isomorphism of $M_{1}$ with $M_{2}$.

We can now prove:

Lemma 1.13. $T_{\mu}$ is consistent, complete and strongly minimal.

Proof. To check that $T_{\mu}$ is consistent we only have to show that $\mathcal{U}$ of Corollary 1.9 satisfies the axioms. By self-sufficient amalgamation everything is clear, except possibly the the case where $B \subseteq \mathcal{U}$ is not self-sufficient and we are given $B \leq C$ to embed into $\mathcal{U}$. Let $\bar{B}:=\bigcap\left\{B^{\prime}: B \subseteq B \leq \mathcal{U}\right\}$ and $\bar{C}=C \oplus_{B} \bar{B}$. If $\bar{C} \in \overline{\mathcal{C}}_{\mu}$ and there exists $X \subseteq \bar{C}$ (realized in $\mathcal{U}$ ) such that $X \cap \bar{B} \not \leq X$ then $X \cap B \not X \cap C$ (because for all $c \in \bar{C} \backslash \bar{B}$ we have $d_{0}(c / \bar{B})=d_{0}(c / B)$ ), and there is nothing to do. If for all $X \subseteq \bar{C}$ realized in $\mathcal{U}$ we have $X \cap \bar{B} \leq X$ we can easily conclude by self-sufficient amalgamation.

By Claim 1.12 to show that $T_{\mu}$ is complete we only have to make sure that the atomic type of the constants is determined and that $\bigcup a_{i} \leq \mathcal{U}$. This follows from the axiom that $d_{0}(A) \geq 0$ for all finite $A \subseteq \mathcal{U}$.

It remains to check that $T_{\mu}$ is strongly minimal. We have to show that if $M=T$ is saturated and $A \subseteq M$ is small then there exists a unique 1-type with infinite orbit under $\operatorname{Aut}(M / A)$. We may assume that $A \leq M$ (since defining $\bar{A}$ as above, $\bar{A} \subseteq \operatorname{acl}(A))$. For $b_{1}, b_{2} \in M$ such that $d\left(b_{i} / M\right)=1$ we have by Claim 1.12 that $t p\left(b_{1} / A\right)=t p\left(b_{2} / A\right)$ so we only have to show that if $d(b / A)=0$ then $b \in \operatorname{acl}(A)$. Let $\bar{b}$ minimal such that $b \in \bar{b}$ and $d_{0}(\bar{b} / A)=0$, then $\bar{b} / F$ is minimally simply prealgebraic for some finite $F \subseteq A$, and therefore has at most finitely many disjoint 
realizations in $M$. By the minimality of $\bar{b} / A$ and the assumption that $A \leq M$ any two realizations of $a t p(\bar{b} / A)$ must be disjoint, which implies that $a t p(\bar{b} / a)$ and therefore $t p(\bar{b} / A)$ has only finitely many realizations in $M$, as required.

Before we proceed to to showing that $T_{\mu}$ has the properties we want some comments on the construction itself are in place. First, it follows immediately from the axiomatization that $\left.T_{\mu}\right|_{S}$ corresponds to the construction (of [3]) with $\left(S, d_{0}, \mu\right)$. Hence $\left.T_{\mu}\right|_{S}$ has DMP, whereas $T_{\mu}$ clearly doesn't. Since in both theories $\operatorname{acl}(A)=\{a: d(a / A)=0\}$ it follows $T_{\mu}$ is a non-trivial acl-preserving expansion of $\left.T_{m u}\right|_{S}$.

We now turn to proving satisfies the main property we are interested in:

Lemma 1.14. $T_{\mu}$ is not a finite cover of a theory with DMP (in particular $T_{\mu}$ does not have DMP).

Proof. Let $E$ be any definable equivalence relation with finite classes. We show that the induced structure $M^{*}=M / E$ does not have DMP. Denote $S^{*}=S / E$ and $R_{i}^{*}=R_{i} / E$. For $a \in M$ denote $S_{a}:=S\left(a, x_{1}, x_{2}\right)$. We show that $R_{i}^{*}$ splits $S_{a_{i}}^{*}$ into two infinite sets for almost all $i$. First, it is clear that $r k\left\{S_{a_{i}}^{*} \cap R_{i}^{*}\right\} \geq 1$ (for if $a_{i}^{*}=$ $a_{i} / E$ then for all generic $\left.\left(b_{1}, b_{2}\right) \in S_{a_{i}}^{*} \cap R_{i}, M_{2}^{*} \models S^{*}\left(a_{i}^{*}, b_{1}^{*}, b_{2}^{*}\right) \wedge R_{i}^{*}\left(b_{1}^{*}, b_{2}^{*}\right)\right)$. So it will be enough to show that if $b$ is a generic element (over $C$, the set of parameters used to define $E$ ) and $\left(b_{1}, b_{2}\right) \in S_{b}$ is a generic element in the corresponding fiber then $M \models S\left(b, b_{1}^{\prime}, b_{2}^{\prime}\right)$ for $b_{i}^{\prime} E b_{i}$ iff $b_{1}=b_{1}^{\prime}$ and $b_{2}=b_{2}^{\prime}$. Assume not then (assuming without loss that $C \leq M$ ) we get that $d\left(b_{1}^{\prime} / C b_{1}\right)=0$ (for $E$ has finite classes and algebraic closure in $M_{2}$ corresponds to the relation $d(a / B)=0$ ), and let $B \supseteq b_{1}^{\prime}$ be a minimal finite set such that $d_{0}\left(B / C b_{1}\right)=0$. Because, as above, $b_{2} \downarrow b_{1}$ necessarily $b_{2}, b_{2}^{\prime} \notin B$ and therefore $d_{0}\left(B / C b_{1}, b_{2}^{\prime}, b\right)<0$. We show that this cannot be:

$$
d_{0}\left(B / C b_{1}, b_{2}^{\prime}, b\right)=d_{0}\left(B b_{1} / C b_{2}^{\prime}, b\right)
$$

so $d_{0}\left(B b_{1} / C b_{2}^{\prime}, b\right)<0$, and so $d_{0}\left(B b_{1} b_{2}^{\prime} / C b\right) \leq 0$ but this implies that $b_{1} \in \operatorname{acl}(b)$, a contradiction.

But for generic $a^{*}$ the set $S_{a^{*}}^{*}$ is strongly minimal so no DMP follows by strong minimality of $T_{\mu}^{*}$.

More generally we can prove:

Lemma 1.15. $T_{\mu}$ has no definable (with parameters) equivalence relations (on singletons) with infinitely many classes with more than one point.

Proof. Assume that $E$ is a definable equivalence relation contradicting the statement of the lemma. Let $C \leq M$ be such that $E$ is defined over $C$, and choose $b$ any element of an $E$-class generic over $C$. Note that for any $b^{\prime} \models E(x, b)$ we have $b^{\prime} \in \operatorname{acl}(C b)$. It follows that $d\left(b^{\prime} / C b\right)=0$. As $b$ is generic over $C$ and $E$ is non-trivial there exists $b^{\prime} \neq b$ as above, and for any such $b^{\prime}$ there exists $B^{\prime} \ni b^{\prime}$ such that $B^{\prime} / C b$ is simply pre-algebraic. Since $\mu\left(B^{\prime} / C b\right) \geq 2$ there exist disjoint $B_{1}, B_{2}$ such that $a t p\left(B^{\prime} / C b\right)=a t p\left(B_{i} / C b\right)$ (this uses also the third bullet of the axiomatization of $T_{\mu}$ which assures that since $C b$ is self-sufficient the number of disjoint solutions of $\operatorname{atp}\left(B^{\prime} / F\right)$ is precisely $\mu\left(B^{\prime} / F\right)$ for $F \subseteq C b$ such that $B^{\prime} / F$ is minimally simply pre-algebraic). Since $b$ is generic over $C$ and $C \leq M$ also $C b \leq M$. Hence $t p\left(B_{1} / C b\right)=t p\left(B_{2} / C b\right)$. In particular, there are $b_{i} \in B_{i}$ such that $\models E\left(b_{i}, b\right)$. 
By transitivity of $E$ it follows also that $d\left(b_{2} / C b_{1}\right)=0$ and we choose a minimal set $B_{1,2}$ witnessing it. We show that $d_{0}\left(B_{1} B_{2} B_{1,2} b / C\right)=0$, which will contradict the fact that $b \notin \operatorname{acl}(C)$. By the minimality of $B_{1,2}$ and the fact that $b_{2} \in B_{1,2} \cap$ $B_{2}$ it follows that $d_{0}\left(B_{1,2} / C B_{1} B_{2}\right) \leq 0$ and equality holds iff $B_{1,2} \subseteq B_{1} B_{2}$. If $d_{0}\left(B_{1,2} / C B_{1} B_{2}\right)<0$ then $d_{0}\left(B_{1} B_{2} B_{1,2} b / C\right)=0$, and we have nothing to do. Otherwise it follows that $d_{0}\left(B_{2} / C B_{1} b\right)<0$, and the contradiction is obtained in a similar way.

Examining the proof of the last lemma we see that in precisely the same way can prove the stronger result:

Lemma 1.16. Let $D=T_{\mu}$ and $S \subseteq D^{n}$ a quantifier free definable strongly minimal set. If $E$ is a definable equivalence relation on $S$ with finite classes and $\bar{b}_{1} \in S$ is generic over all the data then there exists $b \in \bar{b}_{1}$ such $b \in \bar{b}_{2}$ for all $\bar{b}_{2}=E\left(\bar{b}_{1}, y\right)$ and $b$ is generic over all the data.

In the last lemma $\bar{b}_{1} \cap \bar{b}_{2}$ means the intersection of the ranges of the tuples $\bar{b}_{1}, \bar{b}_{2}$. Also note that if $C \leq D$ is such that $S, E$ are definable over $C$ then, as $S$ is quantifier free definable, for any $\bar{b} \in S$ generic over $C$ we have that $C \bar{b} \leq D$. Hence the proof of Lemma 1.15 goes through unaltered if instead of taking a generic $b \in D$ (as we did there) we take $\bar{b} \in S$ generic.

The following remark shows that the assumption that $S$ in the previous lemma was quantifier free definable does not really harm the generality.

Remark 1.17. The reason for requiring that $S$ be quantifier free definable is to assure that for $C \leq D$ and generic $b=S$ we have $C b \leq D$. Note, however, that if $S \subseteq D^{n}$ is any strongly minimal subset and $E$ an equivalence relation with finite classes on $S$ then there exists $m \geq 0$, a quantifier-free definable strongly minimal set $S^{\prime} \subseteq D^{n+m}$ and an equivalence relation $E^{\prime}$ with finite classes on $S^{\prime}$ such that $\pi\left(S^{\prime}\right)=S$ and $E^{\prime}$ is the pre-image of $E$ under $\pi$, the projection on the first $n$ coordinates.

Combining Lemma 1.16 with the last remark we get:

Lemma 1.18. $T_{\mu}$ is not bi-interpretable with any strongly minimal theory with $D M P$.

Proof. We easily reduce to proving that if $S$ is a strongly minimal set definable in $T_{\mu}$ then for every infinite equivalence relation $E$ with finite classes $S / E$ does not have DMP. By the last remark this reduces to proving the above for $S$ that is quantifier free definable. Hence, we may take $S, E$ as in lemma 1.16, and adding constants to the language we may also assume them to be $\emptyset$-definable. Assume first that if $\bar{b}_{1} \in S$ is generic and $\models E\left(\bar{b}_{1}, \bar{b}_{2}\right)$ then their ranges differ (i.e. there exists $\left.b \in \bar{b}_{1} \backslash \bar{b}_{2}\right)$. Let $b \in \bar{b}_{1} \cap \bar{b}_{2}$ be as provided by Lemma 1.16. And assume that $b$ is the $i_{1}^{\text {th }}$ element of $\bar{b}_{1}$ and the $i_{2}^{\text {th }}$ element of $\bar{b}_{2}$. Then $\bar{b}_{i}$ is pre-algebraic over $b$. If $\varphi_{i_{j}}$ is the formula isolating $\operatorname{atp}\left(\bar{b}_{i} / b\right)$ then it also isolates (in its rank) $t p\left(\bar{b}_{i} / \bar{b}_{3-i}\right)$ whence for all $\bar{b}_{i}^{\prime}=\varphi_{i_{j}}$ we have $\models E\left(\bar{b}_{i}^{\prime}, b_{3-i}\right)$. We obtained: if $\bar{b}_{1} \cap \bar{b}_{2}=\{b\}$ and $b$ is the $i_{1}^{\text {th }}$ element of $\bar{b}_{1}$ and the $i_{2}^{\text {th }}$ element of $\bar{b}_{2}$ then $E\left(\bar{b}_{1}, \bar{b}_{2}\right)$ iff $\bigwedge_{1,2} \bar{b}_{i}=\varphi_{i}(x, b)$. Repeating the same process for all possible $i, j$ we get a complete description of $E$. We leave it as an exercise to check that in that case $S / E$ has no DMP.

We now have to check the case that for some $\bar{b}_{1} \in S$ generic and $\bar{b}_{2} \models E\left(\bar{b}_{1}, y\right)$ the ranges of the two tuples agree. Let $\tau$ be a permutation such that $\tau\left(\bar{b}_{1}\right)=\bar{b}_{2}$ 
then $E$ is clearly preserved under the action of the group generated by $\tau$. So let $G$ be the maximal permutation group which acts on the classes of $E$. Let $E_{G}$ be the equivalence relation $E_{G}\left(\bar{b}_{1}, \bar{b}_{2}\right) \Longleftrightarrow \bar{b}_{1}=\tau \bar{b}_{2}$ for some $\tau \in G$. If we quotient out by $E_{G}$ we are in a situation very similar to the one treated above, and the claim follows, more or less as above.

Remark 1.19. Note that the theory $T_{\mu}^{S}:=\left.T_{\mu}\right|_{S}$ is precisely the theory constructed in [3] (for a $\mu$-function satisfying some additional requirements). Therefore $T_{\mu}^{S}$ is strongly minimal with DMP and

$$
a \in \operatorname{acl}_{T_{\mu}^{S}}(A) \Longleftrightarrow d(a / A)=0 .
$$

Since $d(a / A)=0$ depends on $S$ alone in $T_{\mu}$ it follows that the latter is a non-trivial acl-preserving expansion of $T_{\mu}^{S}$.

It may be worth mentioning that the above example indeed does not have DMP but every reduct thereof to a finite sub-language does. It seems, however, that this can be remedied rather easily. Instead of requiring that $\mu$ depends only on $\left.L\right|_{S}$ we allow $\mu$ to depend on $R_{i}$ as well (subject to the obvious requirement that if $A_{a}, \ldots A_{k}$ are all the possible specialization of some $\hat{L}$-finite structure $A, B / A$ is minimally simply pre-algebraic and $\operatorname{atp}_{S}\left(B_{i} / A_{i}\right)=\operatorname{atp}(B / A)$ then $\left.\sum_{i} \mu\left(B_{i} / A_{i}\right)=\mu(B / A)\right)$. If $\mu$ is chosen generically enough, then $R_{i}$ will be reconstructible from $\left.T_{\mu}\right|_{S}$. In the following paragraphs we sketch a different construction which gives the same result. Our construction is a variant of the construction of [1] in which we integrate the ideas used above.

We work in a two sorted language, the sorts being $S, E$ in a language with two function symbols $L=\{f, \pi\}$. Let $T$ be the theory asserting that $\pi: S \rightarrow E$ has infinite fibers; $f: S \rightarrow S$ preserves the fibers of $\pi$ and is generically 1:1, but for each prime $p \in \mathbb{N}$ there is a unique special $p_{1}$-fiber on which either $f^{p}(x)=x$ or $f^{k}(x) \neq f^{j}(x)$ for all $j \neq k \in \mathbb{Z}$, and both cases occur infinitely often. For convenience we will add constants $s_{p} \in E$ such that $\pi^{-1}\left(s_{p}\right)$ is the special $p$-class. These constants will be used just to simplify the construction, then they will be discarded. We leave it as a simple exercise to check that $T$ is a complete $\omega$-stable theory of rank 2 with weak DMP (see [1]) for the definition).

We add a new sort $D$ and a binary relation $R \subseteq S \times D$. Define for a finitely generated $L \cup\{R\}$ structure $A$

$$
d_{0}(A)=\operatorname{MR}(A \backslash D)+|D \cap A|-|R(A)| .
$$

We define $A \leq B$ as usual, with the additional requirement that $A$ is a substructure. We say that $B / A$ pre-algebraic if:

(1) $B / A$ is finitely generated.

(2) $A \leq B$ and $d_{0}(B / A)=0$. If in addition

(3) $B / A$ is minimal such that the above hold we say that $B / A$ is simply prealgebraic.

(4) If also $A$ is minimal then $B / A$ is minimally simply pre-algebraic.

The following is easy:

Claim 1.20. If $B / A$ is simply pre-algebraic there exist finite $b \subseteq B$ and $a \subseteq A$ such that:

- $b$ generates $B$ (over $A$ ).

- $b, a$ are closed under $\pi_{i}$. 
- For any $d \in B$ if $R(d, c)$ for some $c \in A$ then $c \in a$ and $d \in B$.

- If $c:=\pi_{1}(d) \in A$ for some $b \in B$ then $c \in a$.

If we require that $b$ is minimal satisfying the above then it is unique, and similarly for $a$.

We leave the proof as an exercise.

Definition 1.21. A finite tuple $b / A$ is (simply) pre-algebraic if the structure it generates is pre-algebraic and $b$ satisfies the conclusions of the last claim.

Remark 1.22. Let $b / A$ be simply pre-algebraic. There exists a unique $a \subseteq A$ such that $b / a$ is minimally simply pre-algebraic. The atomic type of $b / a$ is isolated. We will identify minimally simply-pre algebraic tuples with the (atomic) formulas isolating them. Note also that if $b / a$ is minimally simply pre-algebraic isolated by $\varphi(x, a)$ there exist a formula $\theta \in$ atp $(a)$ such that for all $a^{\prime} \models \theta$ if $b^{\prime}=\varphi\left(x, a^{\prime}\right)$ then $b^{\prime} / a^{\prime}$ is minimally simply pre-algebraic.

Note that the free amalgam of $L \cup\{D\}$ structures can be defined precisely as above.

The following is an immediate adaptation of the Algebraic Amalgamation Lemma of [3] (see also above) to the present context. We state it in a slightly different way.

Lemma 1.23. Let $A=B_{1} \cap B_{2}$ be $L \cup\{D\}$ structures, $A \leq B_{1}$ and $E=B_{1} \oplus_{A} B_{2}$. Let $d \subseteq E$ and $\varphi(x, d)$ be such that $a \models \varphi(x, d)$ implies that $a / d$ is minimally simply algebraic. There exists a natural number $N$ depending on $\varphi$ (but not on $B_{1}, B_{2}$ ) such that for any $d \subseteq E$ one of the following holds:

(1) $d \subseteq B_{2}$.

(2) There exists a set $X \subseteq B_{2}$ such that $X \cap A \not \leq X$ and $B_{1}$ contains an isomorphic copy of $X$.

(3) There are at most $N$ disjoint realizations of $\varphi(x, d)$ in $E$.

We are now in a situation very much like the the one we encountered in the first construction of this paper. To see this, note that if $\bar{b} / \bar{a}$ is minimally simply pre-algebraic, $\theta(\bar{a})$ isolates the atomic type of $\bar{a} / \emptyset$ and $\varphi(\bar{x}, \bar{y})$ isolates the atomic type of $\bar{b} / \bar{a}$ then for any $\bar{a}^{\prime}=\theta$ there are at most $2^{|\bar{b}|}$ different atomic types in $\varphi\left(\bar{x}, \bar{a}^{\prime}\right)$ which are not outright algebraic (i.e. of negative relative pre-dimension). Ultimately, this follows from the fact that $T$ has weak DMP, but this can be easily verified directly, by checking that for any such $\bar{a}^{\prime}, \bar{b}^{\prime} \models \varphi\left(\bar{x}, \bar{a}^{\prime}\right)$, and any $b \in \bar{b}^{\prime}$ such that $\pi\left(b^{\prime}\right)=s_{p} \in \bar{a}^{\prime}$, deciding whether or not $f^{p}\left(b^{\prime}\right)=b^{\prime}$ or not determines a complete non-algebraic atomic type. Moreover, all possible such completions are admissible. This allows us to define $c(\bar{b} / \bar{a})$ exactly as above.

Finally, it is easy to check that if $\bar{b} / \bar{a}$ is minimally simply pre-algebraic, and $s_{p} \in \bar{a}$ then there a minimally simply pre-algebraic couple $\bar{b}^{\prime} / \bar{a}^{\prime}$ such that $s_{p} \notin \bar{a}^{\prime}$ and $\bar{b} / \bar{a}$ is a specialization of $\bar{b}^{\prime} / \bar{a}^{\prime}$. Call a minimally simply pre-algebraic couple $\bar{b} / \bar{a}$ stationary if $s_{p} \notin \bar{a}$ of any prime $p \in \mathbb{N}$. Call an atomic $\varphi(\bar{x}, \bar{y})$ stationary if it is minimally simply pre-algebraic and is satisfied by some stationary couple. It is standard book-keeping to enumerate (in a non-redundant way) stationary formulas so that every stationary couple satisfies precisely one of them. From this point on, we can proceed in choosing $\mu$ satisfying precisely the same requirements as we did above, and proceed with the construction accordingly, using Lemma 1.23. 


\section{DMP AND GENERIC AUTOMORPHISMS}

Let $T$ be any strongly minimal theory with QE, and $T_{\sigma}:=T \cup\{\sigma$ is an automorphism $\}$. Denote by $T A$ the model companion of $T_{\sigma}$ if it exists. In [4] the following is shown:

Proposition 2.1. Let $T$ be a strongly minimal theory, $M=T$ a saturated model. Assume that for some definable equivalence relation $E$ with finite classes $T h(M / E)$ has DMP. Then TA exists iff already T has DMP.

In their paper Kikyo and Pillay conjecture that indeed this is true in general, i.e. that for strongly minimal theories DMP is equivalent to the existence of $T A$, and remark that if it were true that every strongly minimal set is a finite cover of a strongly minimal set with DMP then the conjecture will follow. We show that the conjecture is true in general. We will use:

Lemma 2.2. Let $G$ be a finite group, $H<G$ a subgroup such that $H$ represents every conjugacy class of $G$ (i.e. for every $\tau \in G$ there exists $\pi \in G$ such that $\pi \tau \pi^{-1} \in H$ ) then $H=G$.

Proof. By assumption $G=\bigcup H^{G}$. Now we compute:

$$
|G|=\left|\bigcup H^{G}\right| \leq(|H|-1)|G / H|+1=|G|-|G / H|+1 \leq|G|
$$

and equality holds iff $H=G$.

We will use the following characterization of DMP which already appears (somewhat implicitly) in [2]:

Lemma 2.3. Let $T$ be a strongly minimal theory, then the TFAE:

(1) $T$ has DMP.

(2) Let $P:=P(a)$ be a strongly minimal set, $G$ a finite group acting regularly and definably on $P, D$ a strongly minimal set definable over $\emptyset$ and $f: P \rightarrow$ $D$ a definable map such that the fibers of $f$ are the orbits of $G$ (except finitely many). Assume that:

(*) For almost all $G$-orbits on $P$ the opposite action of $G$ on the orbit is automorphic

Then there exists a formula $\varphi \in t p(a)$ such that for all $a^{\prime}=\varphi\left(^{*}\right)$ is true of $P\left(a^{\prime}\right)$.

Proof. If $T$ does not have DMP then (2) of the lemma does not hold: from [2] we know that NDMP is witnessed by a strongly minimal set $P(a)=\psi(x, a)$. Assume wlog that for a generic $b \models \psi(x, a), b=\left(b_{1}, \ldots, b_{n}\right), b_{1} / a$ is strongly minimal and $b \in \operatorname{acl}\left(a b_{1}\right)$. Let $\pi_{1}: P(a) \rightarrow D$ (where $D$ is $x=x$ ) be the projection onto the first coordinate. There is no harm in assuming that for all $b^{1}, b^{2} \in P(a)$ if $\pi_{1}\left(b^{1}\right)=\pi_{2}\left(b^{2}\right):=b_{1}$ then there exists some $\sigma \in \operatorname{Sym}(n)$ such that $\sigma(1)=1$ and $\sigma\left(b^{1}\right):=\left(b_{\sigma(1)}^{1}, \ldots, b_{\sigma(n)}^{1}\right)=b_{2}$. Furthermore, we may assume that for almost all $d \in D,\left|\pi^{-1}(d)\right|=m$ for some constant $m \in \mathbb{N}$. Finally, choose $d \in D$ generic over $a$ and let

$$
G=\left\{\sigma \in \operatorname{Sym}(n-1):\left(d, b_{2}, \ldots, b_{n}\right) \in P(a) \Rightarrow\left(d, b_{\sigma(2)}, \ldots, b_{\sigma(n)}\right) \in P(a)\right\}
$$

Since we have chosen $d$ generic over $a$ it follows that $G$ acts regularly (and obviously definably) on almost all the fibers of $\pi_{1}$. Note also that it cannot be that $|G|=1$, 
as this would contradict the assumption that $P(a)$ is a counter example to DMP. It is now immediate to check that by NDMP this is a counter example for (2).

For the other direction, assume that $T$ has DMP and that $P(a), D, f$ and $G$ are as in $(*)$. We have to show that the conclusion of (2) holds. Choose $\theta \in t p(a)$ such that for all $a^{\prime} \models \theta, P\left(a^{\prime}\right)$ is strongly minimal and we may further restrict $\theta$ so that for all $\left(b_{1}, \ldots, b_{n}\right) \in P\left(a^{\prime}\right), b_{1}$ is interalgebraic with $b_{i}$ for all $i$ and that $\left(b_{\sigma(1)}, \ldots, b_{\sigma(n)}\right) \in P\left(a^{\prime}\right) \Longleftrightarrow \sigma \in G$ (we may of course think of $G$ as a permutation group, in accordance to its action on a generic fiber of $P(a)$ ), then by strong minimality of $P\left(a^{\prime}\right)$ the opposite action of $G$ on the fibers of $P\left(a^{\prime}\right)$ is automorphic almost everywhere, and we are done.

Now we are ready to prove:

Theorem 2.4. Let $T$ be a strongly minimal theory, then $T A$ exists iff $T$ has DMP.

Proof. The proof that if $T$ has DMP then $T A$ exists appears in [4]. For the other direction suppose that $T A$ exists. Let $P(a)$ be strongly minimal, we have to find $\theta \in t p(a)$ such that $a^{\prime} \models \theta \Rightarrow P\left(a^{\prime}\right)$ is strongly minimal. As in the direction " $\Rightarrow "$ of the previous lemma we can define a map $\pi_{1}: P(a) \rightarrow D$ with finite fibers, and assume that any two elements in a generic fiber differ only by a permutation, and let $G$ be the corresponding permutation group. Let $\theta \in t p(a)$ be such that:

- For all $a^{\prime}=\theta, P\left(a^{\prime}\right)$ has rank 1 ;

- If $\left(b_{1}, \ldots, b_{n}\right) \in P\left(a^{\prime}\right)$ then for all $i, b_{i}$ is interalgebraic with $b_{1}$ and

- For all $\tau \in \operatorname{Sym}(n-1),\left(b_{1}, b_{\tau(2)}, \ldots, b_{\tau(n)}\right) \in P\left(a^{\prime}\right) \Longleftrightarrow \tau \in G$. In other words, $G$ acts regularly (and, of course, definably) on all the fibers of $\pi_{1}\left(a^{\prime}\right)$ for all $a^{\prime}=\theta$.

Let $M \models T A$ and let $a^{\prime} \in \theta(M)$ be such that $\sigma$ fixes $a^{\prime}$ (pointwise). For each $\tau \in G$ there exists $b_{\tau}:=\left(b_{1}, \ldots, b_{n}\right) \in P\left(a^{\prime}\right)^{M}$ such that $\sigma\left(b_{1}\right)=b_{1}$ and $\sigma\left(b_{i}\right)=b_{\tau(i)}$ for $i>1\left(b_{\tau}\right.$ exists since $a^{\prime} \models \theta$ implying that $G$ acts automorphically on the fibers of $\pi_{1}$ and using the fact that $M$ is existentially closed we can find $b_{\tau}$ as required).

Finally we show that this $\theta$ satisfies the requirements. Let $a^{\prime}=\theta^{M}$ for a large saturated $M \models T$. Expand $M$ to $M^{\prime} \models T A$ in such a way as to have $\sigma \uparrow_{\operatorname{acl}_{T}\left(a^{\prime}\right)}=\mathrm{Id}$. Let $\tau \in G$ and take $b_{\tau}:=\left(b_{1}, \ldots, b_{n}\right) \in P\left(a^{\prime}\right)$ as promised in the previous paragraph (i.e. $\sigma\left(b_{1}\right)=b_{1}$ and $\sigma\left(b_{i}\right)=b_{\tau(i)}$ for $\left.i>1\right)$, and let $\left(d_{1}, \ldots, d_{n}\right)$ be any generic of $P\left(a^{\prime}\right)$. Then $t p\left(d_{1} / \operatorname{acl}_{T}\left(a^{\prime}\right)\right)=t p\left(b_{1} / \operatorname{acl}_{T}\left(a^{\prime}\right)\right)$ (both being generics of $D$ ) so there is some $\alpha \in \operatorname{Aut}\left(M / \operatorname{acl}\left(a^{\prime}\right)\right)$ with $\alpha\left(b_{1}\right)=\alpha\left(b_{2}\right)$ then $\alpha^{-1} \tau a$ acts automorphically on the fiber above $d_{1}$. since $\alpha$ was an automorphism, this implies that the subgroup of $G^{o p}$ acting by automorphisms on the fiber of $d_{1}$ meets every conjugacy class of $G$, so by what we have already shown, this implies that $G^{o p}$ acts by automorphism on the fiber of $d_{1}$. By the previous lemma this implies that $T$ has DMP.

Acknowledgment: The authors wish to thank Frank Wagner for pointing out a gap in the original proof of lemma 2.2 and Gregory Cherlin for supplying them with its elementary proof given in the text.

\section{REFERENCES}

[1] A. Hasson. Interpreting structures of finite Morley Rank in strongly minimal structures. Preprint (2003). 
[2] E. Hrushovski. Strongly minimal expansions of algebraically closed fields. Israel Journal of Mathematics 79, 129-151 (1992).

[3] E. Hrushovski. A new strongly minimal set. Annals of Pure and Applied Logic 62, 147-166 (1993).

[4] H. Kikyo And A. Pillay. The definable multiplicity property and generic automorphisms. Annals of Pure and Applied Logic 106, 263-273 (2000).

* Mathematical Institute, Oxford University, Oxford, UK

E-mail address: hasson@maths.ox.ac.uk

URL: http://www.maths.ox.ac.uk/ ^hasson/

†Einstein Institute of Mathematics, The Hebrew University of Jerusalem, Jerusalem, ISRAEL

E-mail address: ehud@math.huji.ac.il 